\title{
Ultra-High-Resolution Method for Solid-State Image Sensors Using Multiple-Step Swing and Wobbling Operation
}

\author{
（多段スウィング・ウォーブリング動作による固体イメージセンサの超高解像度化方式）
}

Nozomu Harada $^{\dagger}$ (member) and Okio Yoshida ${ }^{\dagger \dagger}$

\begin{abstract}
An ultra-high-resolution method using a multiple-step swing and wobbling operation is described for improving the resolution of solid-state image sensors. The method is based on the periodical shifting of the device to form multiple pixels. By analyzing the operation of this method, the optimum operation conditions were derived for achieving high resolution with low aliasing and low field flicker phenomena. Also described is a multiple-step wobbling operation with triangular waves that achieves high resolution with even lower aliasing and a wider operation range. Suitable display conditions of the multiple-step swing imaging signals were derived for achieving high resolution display images.
\end{abstract}

Key words : Solid state image sensor, Display device, Resolution, Nyquist frequency, Aliasing, Fourier transform

\section{Introduction}

Solid-state image sensors are key devices in various imaging systems. Resolution is one of the most important aspects of the performance of these devices. The improvement of resolution has been strongly and continuously required. It has been achieved simply by increasing pixel density and by expanding image area. The increase in pixel density unfortunately leads to decreases of sensitivity and dynamic range. The expansion of image area leads to larger, heavier and more costly systems.

To address this high-resolution requirement without deterioration of other image characteristics, the authors successfully developed a Swing CCD image sensor using a CCD chip shifting operation and realized double resolution enhancement ${ }^{12)}$. A swing operation using an incident image shifting instead of the CCD chip shifting can achieve the same resolution enhancement effect ${ }^{3)}$. The authors analyzed the double resolution enhancement effect for both the CCD chip shifting and the incident image shifting ${ }^{4)}$. Higher resolution enhancement is required for the Swing CCD image sensor.

\footnotetext{
Received March 28, 2001, Revised September 11, 2001,

Acceped October, 4, 2001

$†$ Toshiba Electronic Engineering Corporation, Display Design Service Center ( ₹ 1-9-2 Hatara-cho, Fukaya-shi, Saitama, 366-8510, Japan, TEL 048-574-5607)

$\dagger \dagger$ Josai International University, Depertment of Media and Culture Studies (1 Kiumei, Toukin-si, Tiba, 283-8555, Japan, TEL 0475-55-8800)
}

In the present work, we proposed a higher resolution method using a novel multiple-step swing and wobbling operation. The multiple-step swing and wobbling method can realize a higher resolution image with low aliasing and low field flicker phenomena. We analyzed molulation transfer function characteristics and aliasing effects for different pixel parameters and operation modes. Optimum operation conditions for the higher-resolution enhancement are derived by using the analytical results. We also describe an application of the multiple-step swing operation to display devices.

\section{Low Aliasing Condition in Swing Operation}

Fig. 1 shows the swing operation principle for the solidstate image sensor. In this case, the image sensor is supposed to be an interline-transfer CCD, in which a unit pixel is composed of a photosensing area shown by a rectangular aperture shape and a vertical CCD register for signal charge read-out. The vertical CCD register is optically shielded. One frame picture is formed by two field pictures of the field $\mathrm{A}$ and the field $\mathrm{B}$. Stored signal charges during the field $\mathrm{A}$ period are transferred to the vertical CCD register during a vertical blanking period. This signal charge transfer is simultaneously operated in all pixels. At the same time or just after the signal charge transfer, the CCD chip is shifted horizontally to a new position at a half pixel distance. Then, signal charge stor-

(171) 315 


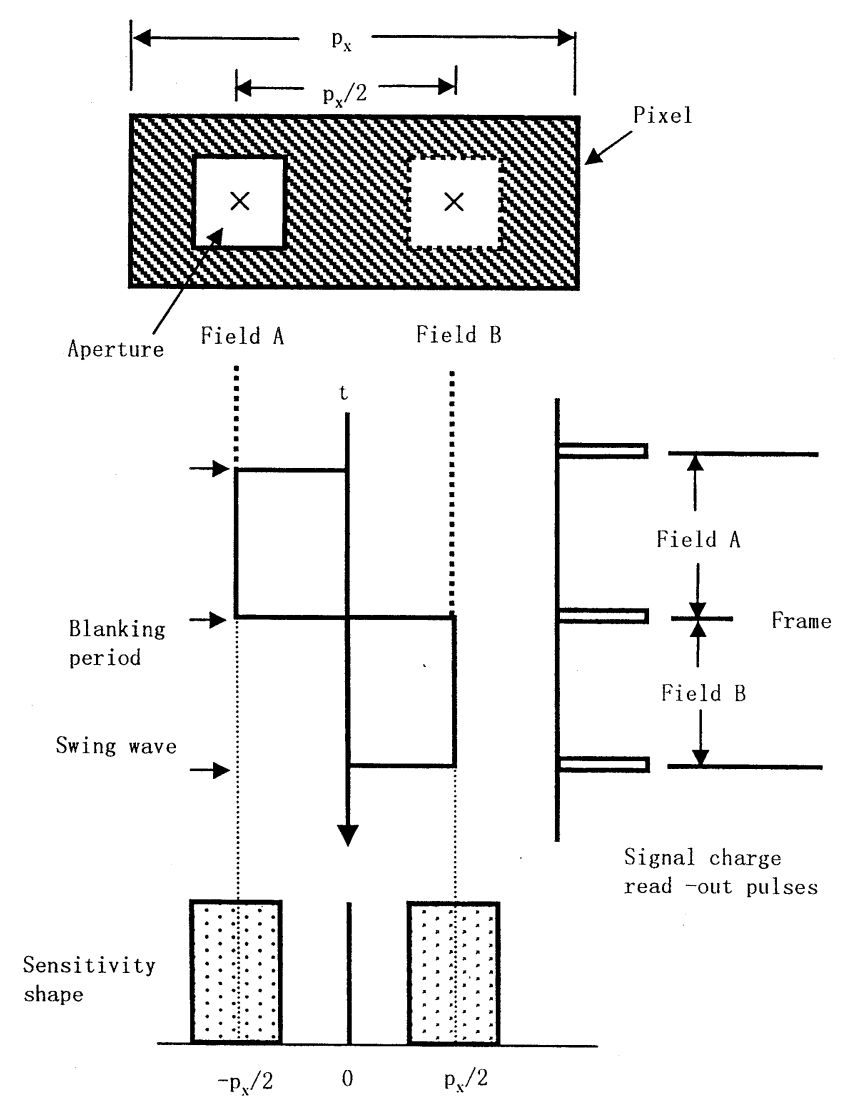

Fig. 1 Swing operation principle for solid-state image sensor.

age for the field $B$ starts at the new position. Thus, the obtained time-shared images with horizontally interlaced signals are re-arranged on a display monitor to produce images achieving double enhanced resolution. As a result, two pixel apertures are formed in the unit pixel of the conventional $\mathrm{CCD}$ by using the swing CCD operation. In the TV system, one frame picture is formed by two field pictures. By the afterimage characteristic of a human's retina, the two field pictures superimposed on a screen are recognized as one picture. The resolution enhancement of the Swing CCD is also realized by the same aferimage effect of human's retina. A Swing CCD camera, adapted to the standard TV format, has been developed 5). Therefore, the swing operation can be applied to not only still imaging systems, but also to motion imaging systems.

A range of the reproducible image exists in the first Brillouin zone. The boundary of the zone is defined as a Nyquist frequency, that is a resolution limit. In the conventional case without the swing operation, the Nyquist frequencies $F_{n x}$ and $F_{n y}$ in $x$-and $y$-directions are given by

$$
F_{n x}=1 / 2 p_{x}, \quad F_{n y}=1 / 2 p_{y}
$$

where $p_{x}$ and $p_{y}$ are pixel pitches in $x$ - and $y$-direction, respectively. When $\mathrm{u}$ and $\mathrm{v}$ are rewritten with the nor- malized frequencies $u^{*}\left(=u / F_{n x}\right)$ and $v^{*}\left(=v / F_{n y}\right)$, respectively, an MTF (Modulation Transfer Function) for a rectangular pixel aperture shape is given by a sinc function ${ }^{6)}$.

$$
\begin{aligned}
\text { MTF } & =\left|\frac{\sin \left(\frac{\pi}{2} \alpha_{x} u^{*}\right)}{\frac{\pi}{2} \alpha_{x} u^{*}} \cdot \frac{\sin \left(\frac{\pi}{2} \alpha_{y} v^{*}\right)}{\frac{\pi}{2} \alpha_{y} v^{*}}\right| \\
& =\mid \operatorname{sinc}\left(\frac{\pi}{2} \alpha_{x} u^{*}\right) \operatorname{sinc}\left(\frac{\pi}{2} \alpha_{y} v^{*}\right)
\end{aligned}
$$

where, we use aperture ratios of $a_{x}\left(=\mathrm{a} / p_{x}\right)$ and $a_{y}$ $\left(=a / p_{y}\right)$ in $x$ - and $y$-directions, respectively. The resolution enhancement technique using the swing operation can be applied to two imaging modes, that is, the progressive and interlacing modes. Fig. 2 shows sampling center arrangement and Nyquist domain shapes without and with the swing operation. In the case of the progressive mode swing operation, the sampling points are arranged in a square lattice pattern with double the number of pixels, resulting in a doubling of the Nyquist domain area in the horizontal direction. Therefore the horizontal Nyquist frequency is doubly enhanced as,

$$
F_{n x}=1 / p_{x}
$$

In the case of the interlacing mode swing operation, the sampling centers are rearranged in a rhombus lattice pattern with the same number of sampling points. The Nyquist domain shape is transferred from a square to a rhombus with the same domain area. The horizontal Nyquist frequency is double that in the case of conventional imaging without the swing operation when the horizontal sampling pitch is larger than the double vertical sampling pitch, $p_{x}>2 p_{y}$. When the horizontal sampling pitch is smaller than the double vertical sampling pitch, the horizontal Nyquist frequency is given as ${ }^{6}$,

$$
\begin{aligned}
F_{n x} & =\frac{p_{x}^{2}+4 p_{y}^{2}}{8 p_{x} p_{y}^{2}} \\
& =\frac{1}{2 p_{x}}\left\{1+\left(\frac{P_{x}}{2 p_{y}}\right)^{2}\right\}\left(\frac{1}{2 p_{x}}<F_{n x}<\frac{1}{p_{x}}\right)
\end{aligned}
$$

This means that the resolution enhancement effect of the swing operation is reduced for CCD with high horizontal pixel density. Considering the vertical resolution, a condition of $p_{x}=2 p_{y}$ is desirable for the resolution enhancement using the interlacing mode swing operation.

The aliasing appears in the lower frequency region due to return of high spatial frequency components of the MTF with mirror-reflectance characteristics at integer multiplication of the Nyquist frequency. For this resolu- 


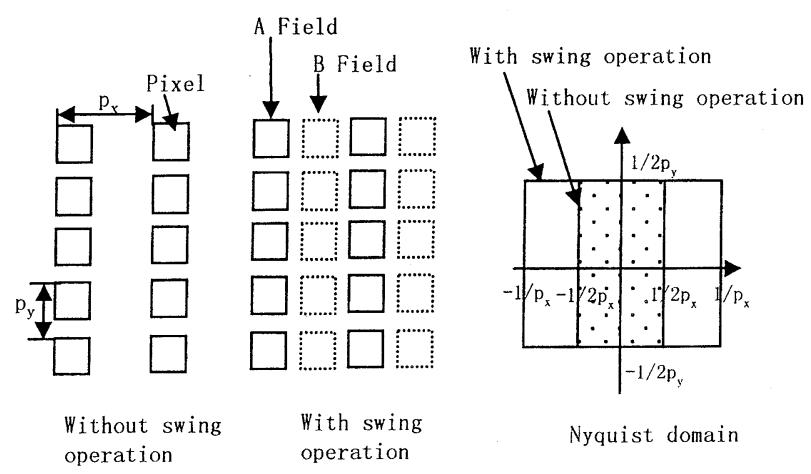

(a) Progressive imaging mode

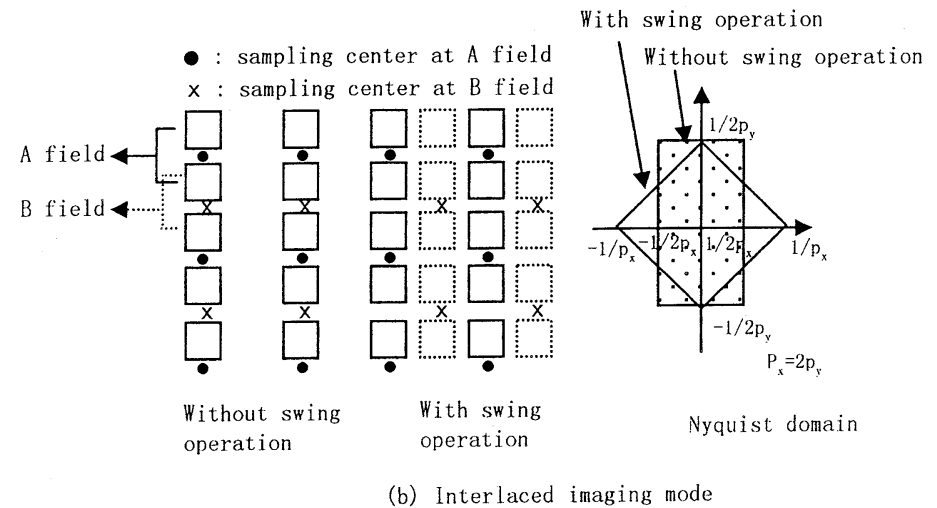

Fig. 2 Sampling center arrangement and Nyquist domain shapes.

tion evaluation, we have newly introduced a ratio of the aliasing amplitude to the MTF inside the Nyquist frequency $^{4)}$. We refer to this ratio as the aliasing rate. The horizontal Nth aliasing rate $A N\left(u^{*}\right)$ for the triangular wave swing operation is given by

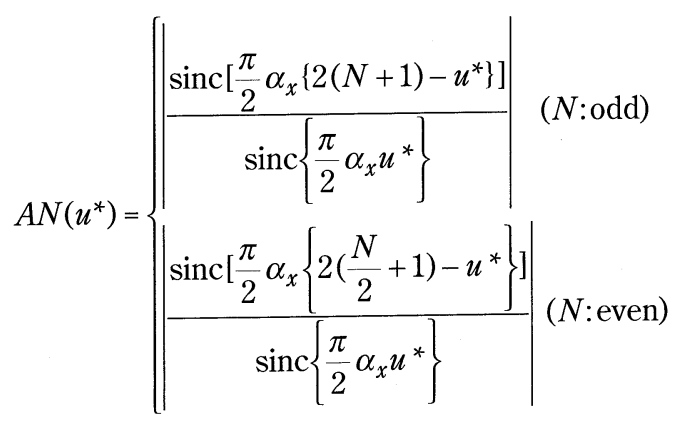

where $N$ is integers expressing the aliasing of the Nth Nyquist region of $N \leqq u^{*} \leqq N+1$.

Fig.3 shows the MTFs and the first aliasing $\quad(N=1)$ characteristic for the rectangular swing operation in the case of $a_{x}=1$. The Nyquist frequency exists at $u^{*}=2$ with the swing operation in comparison with $u^{*}=1$ for the conventional mode without the swing operation. In addition to the resolution limit expansion, a lower MTF at an enhanced Nyquist frequency $u^{*}=2$ leads to a reduction of "aliasing phenomena". When fine repeated pattern is imaged, the aliasing causes a deformed signal called as
Nyquist frequency
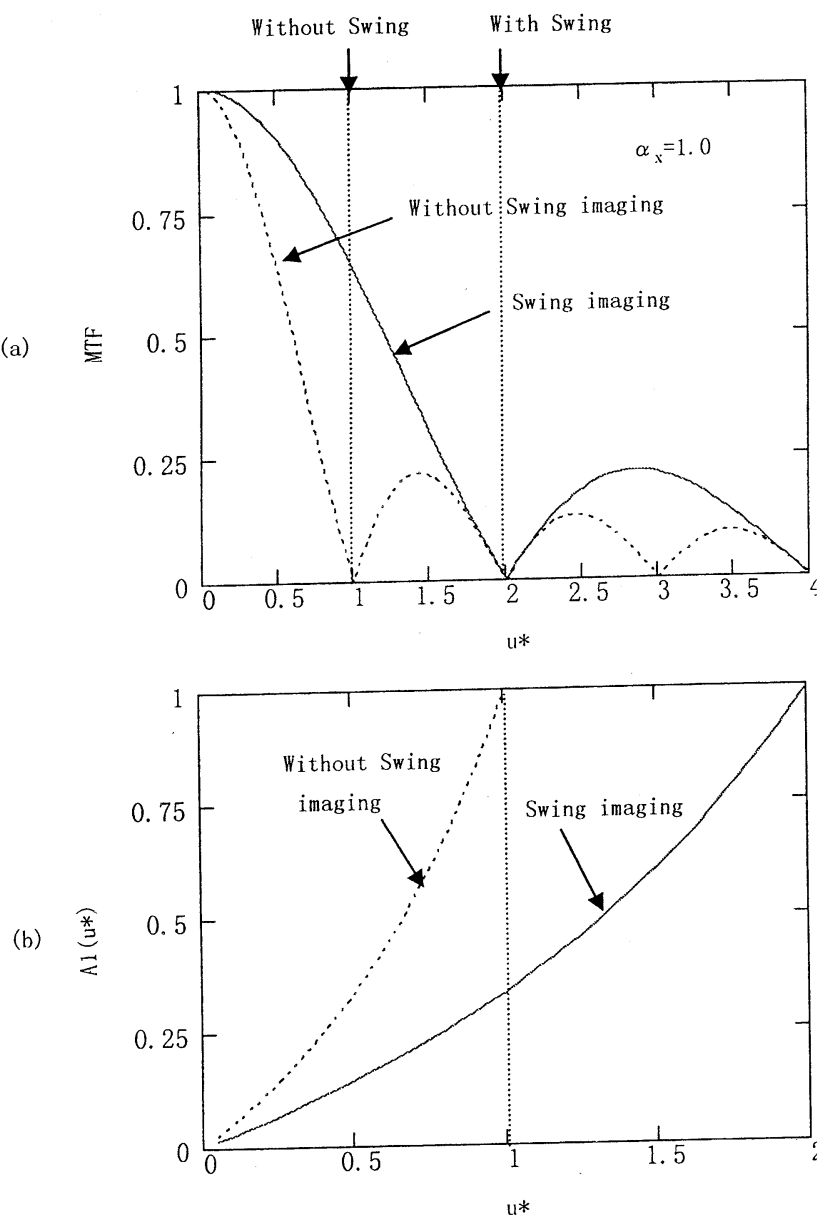

Fig. 3 The MTFs and the first aliasing characteristics for the rectangular swing operation in the case of $\alpha_{x}=1$. 
"moiré" or "beat pattern" in the reproduced picture. An aliasing signal suppression is important in obtaining a high-resolution picture as the resolution limit is reduced if the aliasing occurs near the Nyquist frequency. Further, the aliasing at low frequencies leads substantially to quality degradation of an entire image. Therefore, an MTF design considering the aliasing suppression is required for a higher resolution swing operation. As shown in Fig.3, the aliasing rates for both modes are pinned to zero at $u^{*}=0$ when $a_{x}$ is unity. In particular, in the case of the swing operation, the aliasing signals at $u^{*}=0$ and Nyquist frequency $\left(u^{*}=2\right)$ are doubly pinned to zero. This double aliasing zero pinning also occurs in higher frequency aliasing regions and gives a high resolution picture with a low aliasing phenomena. However the double aliasing zero pinning can not be satisfied for conventional CCDs, with the exception of $\mathrm{CCD}$ with photoconductive layer overlaid structure ${ }^{7)}{ }^{8)}$, because the aperture ratio $a_{\mathrm{x}}$ is not unity. For the requirement of $a_{x}=1$, the wing CCD can expand the aperture ratio by superimposing a wobble at the swung site ${ }^{9)}$, realizing the double aliasing zero pinning condition. For the multiple step mode, the double aliasing zero pinning added to the Nyquist frequency increase is required. Thus, optimum swing and wobbling conditions have to be also derived for the higher-resolution enhancement using the multiple step shifting of CCD.

\section{Four Step Swing Operation}

We derived an optimum condition using a swing operation with two step shifting in which pixel density could be doubled ${ }^{4)}$. Swing operation with more steps is needed for higher-resolution enhancement.

Fig.4 shows MTFs for a four step swing operation with a rectangular wave when $\alpha_{x}$ is varied. In the figure, aliasing characteristics in a double aliasing zero pinning condition are also shown. The Nyquist frequency for the progressive imaging mode is located at $u^{*}=4$. The double aliasing zero pinning is obtained at $a_{x}=0.5$. The MTF in the case of $a_{x}=0.25$ can be adjusted to be zero at the Nyquist frequency $u^{*}=4$ by using an optical or electrical low-pass filter. Therefore, the condition of $a_{x}=0.25$ can also satisfy the condition of the double aliasing zero pinning at $u^{*}=0$ and 4 .

Fig. 5 shows the pixel aperture shifting in the four-step swing operation with a rectangular wave. Four pixel apertures with $p_{x} / 4$ pitch distance are formed in the unit pixel of the conventional device. While, in the swing operation, the image at each field has an aliasing with the Nyquist frequency of $u^{*}=1$. In the four step swing opera-
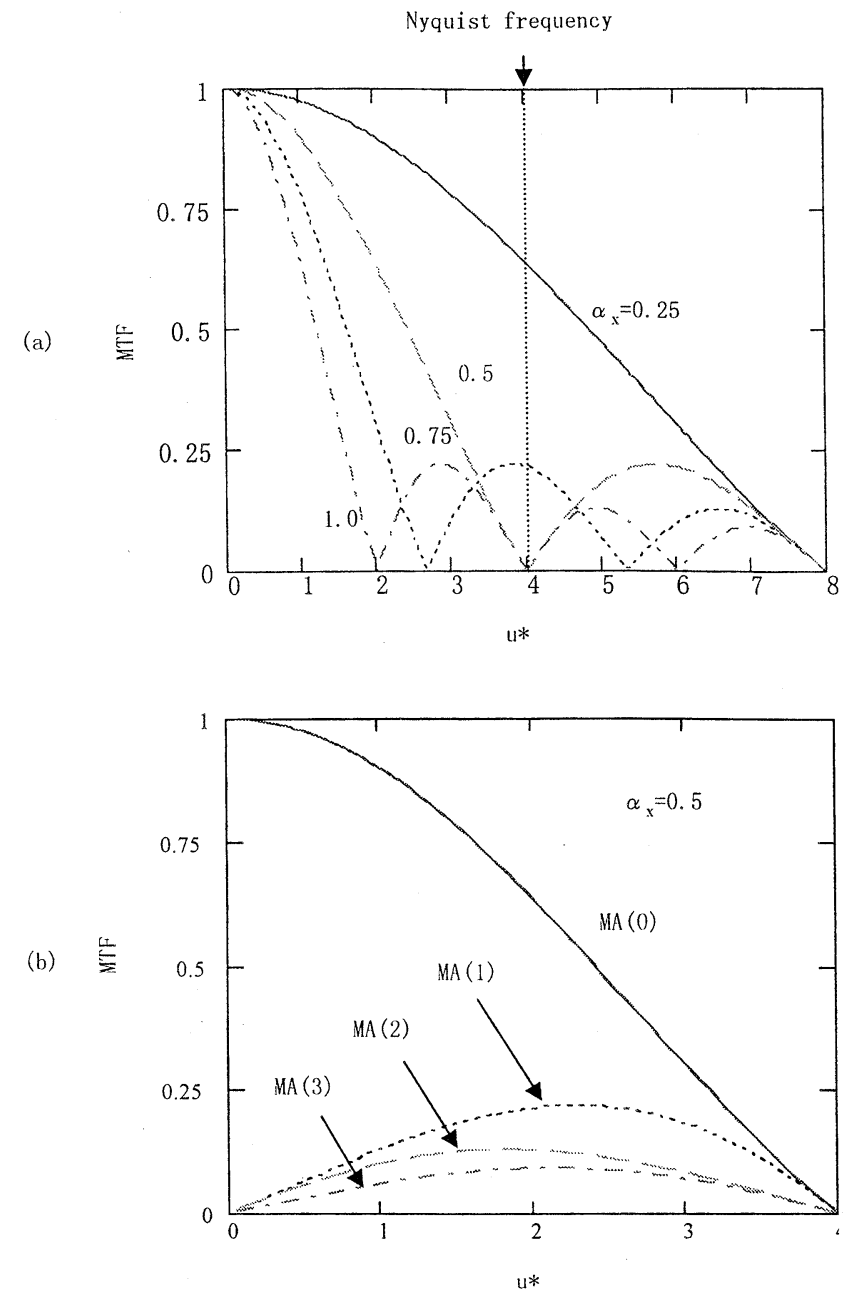

Fig. 4 The MTF characteristics for a four step swing operation with a rectangular wave when $\alpha_{\mathrm{x}}$ is varied.

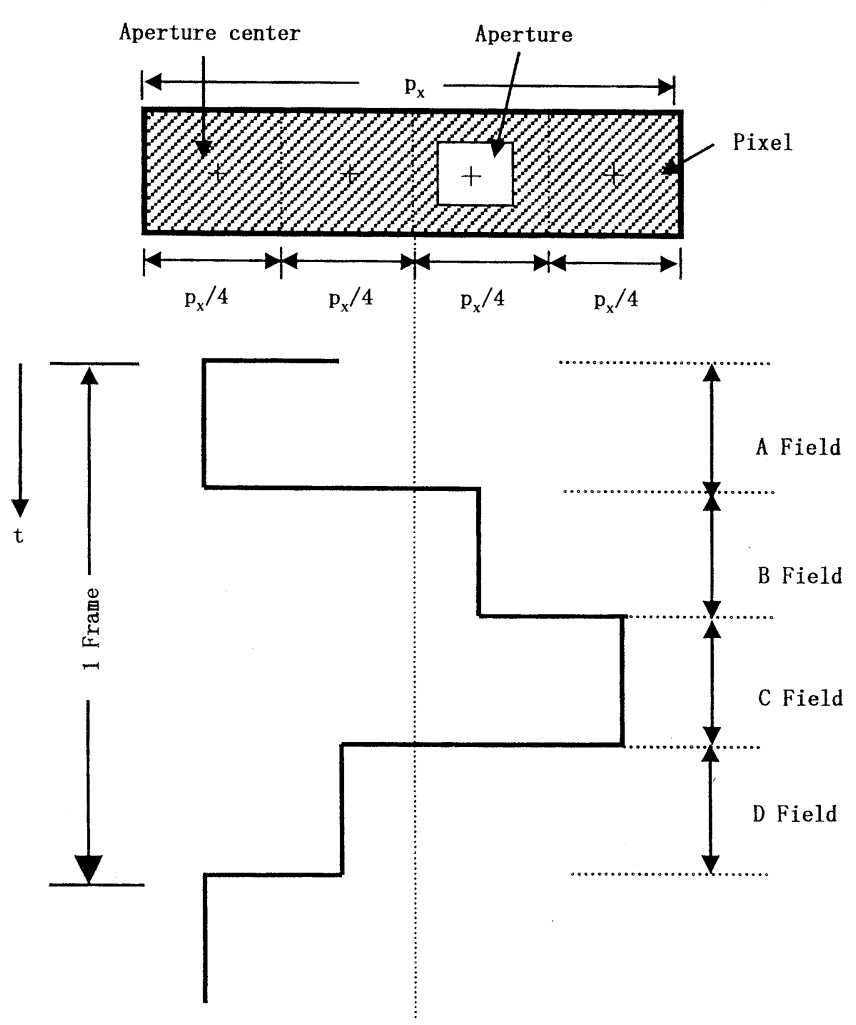

Fig. 5 The pixel aperture shifting in the four step swing operation with a rectangular wave. 

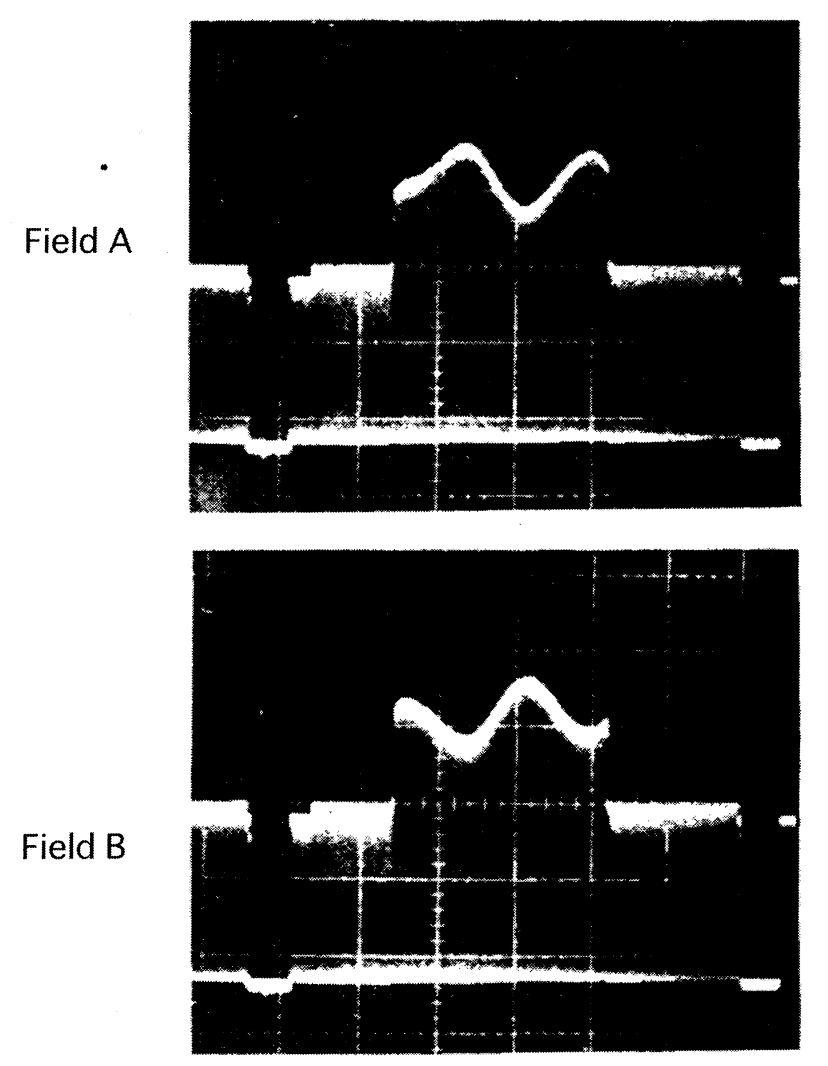

\section{$\mathrm{Y}: 100 \mathrm{mV} / \mathrm{div} \quad \mathrm{X}: 5 \mathrm{~ms} / \mathrm{div}$}

Fig. 6 Line-selected signal waves at the field A and the field B in the case of a vertical black and white bar pattern of around Nyquist frequency produced by a Swing CCD image sensor.

tion, the high aliasing at the individual field is compensated by successive two fields having $180^{\circ}$ phase difference, between the field $\mathrm{A}$ and the field $\mathrm{B}$, and between the field $\mathrm{C}$ and the field D as well. Fig. 6 shows line-selected signal waves at the field $A$ and the field $B$ when a vertical bar pattern at around Nyquist frequency is taken by a Swing CCD image sensor. As shown in the figure, aliasing signals with $180^{\circ}$ phase difference appear at the field $\mathrm{A}$ and the field $\mathrm{B}$. By combining the successive two field signals, the aliasing is compensated for two field periods which are a half of one frame period. When the four-step operation is applied to the TV format, the four pictures in the four fields have to be reformed into two field pictures. Thus, a new field picture is formed by combining the two field pictures of the field $\mathrm{A}$ and the field B. Similarly, the two field pictures of the field $\mathrm{C}$ and the field D are transformed into one field picture. This transformation can be easily made by using a signal processing circuit in the camera system. In this case, the short time aliasing compensation operation gives two field pictures with low aliasing, resulting in reduction of field flicker phenomena caused by high aliasing at each field. In the still imaging, a field flicker appeared on the display also becomes to be a problem. The four pictures of the four fields are transformed to two field pictures for the TV format display. In the display operation, high aliasing in each field leads to a field flicker at a pattern edge with high frequency components. Therefore, the short time aliasing compensation is also suitable for still imaging applications. When the step number is odd, the individual field cannot have a counter field with $180^{\circ}$ spatial phase difference, and so the short time aliasing suppression does not occur. Therefore, the even step number for the multiple-step swing operation is desirable.

An MTF control is realized by a wobbling operation in which wobbling or a vibration is superimposed at swung sites ${ }^{9)}$. The MTF is controlled basically due to a pixel aperture expansion using the wobbling. The aliasing zero pinning at $u^{*}=0$ and 2 can be realized by an electrical MTF control using the wobbling operation. The authors derived previously that a triangular wave wobbling operation realizes high-resolution image with lower aliasing. When wobbling amplitude of triangular wave wobbling operation is $2 L_{w}$, a pixel shape function $f(x)$ is given by ${ }^{4)}$

$$
\begin{array}{r}
f(x)=\frac{Q_{f}}{2 a}\left\{\operatorname{rect}\left(\frac{x}{L_{w t}}\right) \cdot \operatorname{rect}\left(\frac{x-\frac{L_{w t}}{2}}{a}\right)\right. \\
\left.+\operatorname{rect}\left(\frac{x}{L_{w t}}\right) \cdot \operatorname{rect}\left(\frac{x+\frac{L_{w t}}{2}}{a}\right)\right\}
\end{array}
$$

where $Q_{\mathrm{f}}$ and "a" show an integral signal intensity during one field period and a horizontal aperture length, respectively. Then its Fourier transform $F\left(u^{*}\right)$ is given by

$$
F\left(u^{*}\right)=\frac{2 Q_{f}}{a} \operatorname{sinc}\left(\frac{\pi}{2} \alpha_{x} u^{*}\right) \cdot \operatorname{sinc}\left(\pi \beta_{\mathrm{x}} u^{*}\right)
$$

where $\beta_{x}$ is $L_{w t} / p_{x}$, calling a wobbling rate for the triangular wave wobbling. Therefore, the MTF is given by

$$
\operatorname{MTF}\left(u^{*}\right)=\left|\operatorname{sinc}\left(\frac{\pi}{2} \alpha_{x} u^{*}\right) \cdot \operatorname{sinc}\left(\pi \beta_{x} u^{*}\right)\right|
$$

And, the first aliasing rate A1 $\left(u^{*}\right)$ for the four-step triangular wave wobbling operation is given by

$$
A 1\left(u^{*}\right)=\left|\frac{\operatorname{sinc}\left[\frac{\pi}{2} \alpha_{x}\left(8-u^{*}\right)\right]}{\operatorname{sinc}\left(\frac{\pi}{2} \alpha_{x} u^{*}\right)}\right| \cdot\left|\frac{\operatorname{sinc}\left[\pi \beta_{x}\left(8-u^{*}\right)\right]}{\operatorname{sinc}\left(\pi \beta_{x} u^{*}\right)}\right|
$$






Fig. 7 The MTF characteristic of the four step triangular wobbling operation in the case of $\alpha_{X}=0.5$ when $\beta_{X}$ is varied.

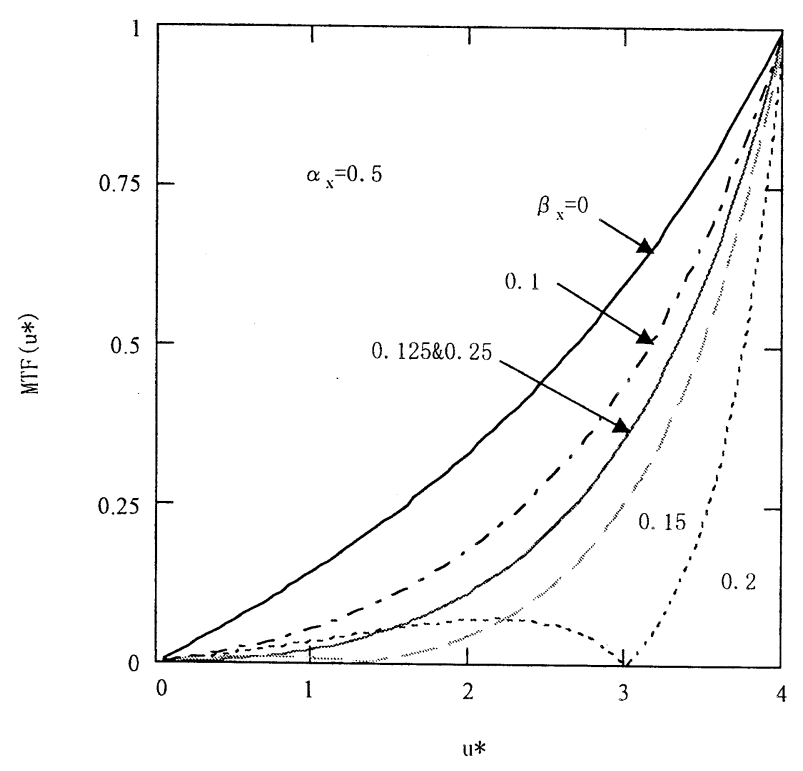

Fig. 8 The aliasing rate characteristic for the four step triangular wobbling operation in the case of $\alpha_{X}=0.5$ when $\beta_{X}$ is varied at less than 0.25 .

Fig.7 shows the MTF characteristic of the four-step triangular wave wobbling operation in the case of $\alpha_{x}=0.5$ when $\beta_{x}$ is varied. when $a_{x}$ is 0.5 , aliasing values at $u^{*}=0$ and Nyquist frequency $u^{*}=4$ are pinned to zero. As shown in this figure, the MTF becomes zero at a frequency less than the Nyquist frequency of $u^{*}=4$ in the case of $\beta_{x}>0.25$. This means that the resolution limit becomes less than $u^{*}=4$. Therefore, it is recommended that $\beta_{x}$ is set to less than 0.25 so as to assure high resolution.

Fig. 8 shows the aliasing rate characteristic in the case of $\alpha_{x}=0.5$ when $\beta_{x}$ is varied at less than 0.25 . A solid line at $\beta_{x}=0$ is the aliasing rate of the rectangular wave swing operation without the wobbling. As shown in this figure, the more $\beta_{x}$ increases at $\beta_{x}<0.125$, the less the aliasing rate monotonously decreases. Therefore, this leads to a condition such that triangular wave wobbling is superior to the rectangular swing operation for the four step swing operation. In the case of $0.15 \leq \beta_{x} \leq 0.25$, the aliasing rate at low frequency region is further suppressed low with the pinning effect at $\mathrm{u}^{*}=0$. The aliasing rate at $\beta_{x}=0.25$ matches that at $\beta_{x}=0.125$. This means that the MTF for $\beta_{x}=0.25$ coincides with that of $\beta_{x}=0.125$ when the MTF for $\beta_{x}=0.125$ is operated so as to be zero at $u^{*}=4$. Also, a similar double aliasing zero pinning can be achieved by setting $\beta_{x}=0.25$ in spite of $a_{x}=0.5$. In this case, a condition with $0.5 \leq \alpha_{x} \leq 1.0$ is more suitable for the higher resolution imaging with lower aliasing. As mentioned above, the double aliasing zero pinning is realized by setting $\alpha_{x}$ and $\beta_{x}$ to appropriate values.

\section{Multiple-Step Wobbling Operation}

A suitable operation condition for a multiple-step wobbling operation is further discussed in this section. An Mstep wobbling operation, in which $\mathrm{M}$ is integer for even numbers, is applied for greater resolution enhancement. An optimum operation condition is obtained by the control of the aperture ratio $a_{x}$ and the wobbling ratio $\beta_{x}$. Requirements for the high resolution imaging with better picture quality are, (1) the double aliasing zero pinning at $u^{*}=0$ and $\mathrm{M},(2)$ the maintaining of the resolution limit at the Nyquist frequency $M$, and (3) the short time aliasing compensation between two successive field.

Fig. 9 shows the $M$-step wobbling operation with a rectangular or a triangular wobbling wave. The CCD is operated in a progressive imaging mode. The sensitivity shapes for image sensor are denoted in a convex for the rectangular wobbling wave and a trapezoid for the triangular wobbling wave.

\subsection{Analysis on Rectangular Wobbling}

The MTF and the $N$-th of aliasing rate characteristics for rectangular wobbling are given by

$$
\operatorname{MTF}\left(u^{*}\right)=\left|\operatorname{sinc}\left(\frac{\pi}{2} \alpha_{x} u^{*}\right) \cdot \cos \left(\pi \beta_{x} u^{*}\right)\right|
$$

and 


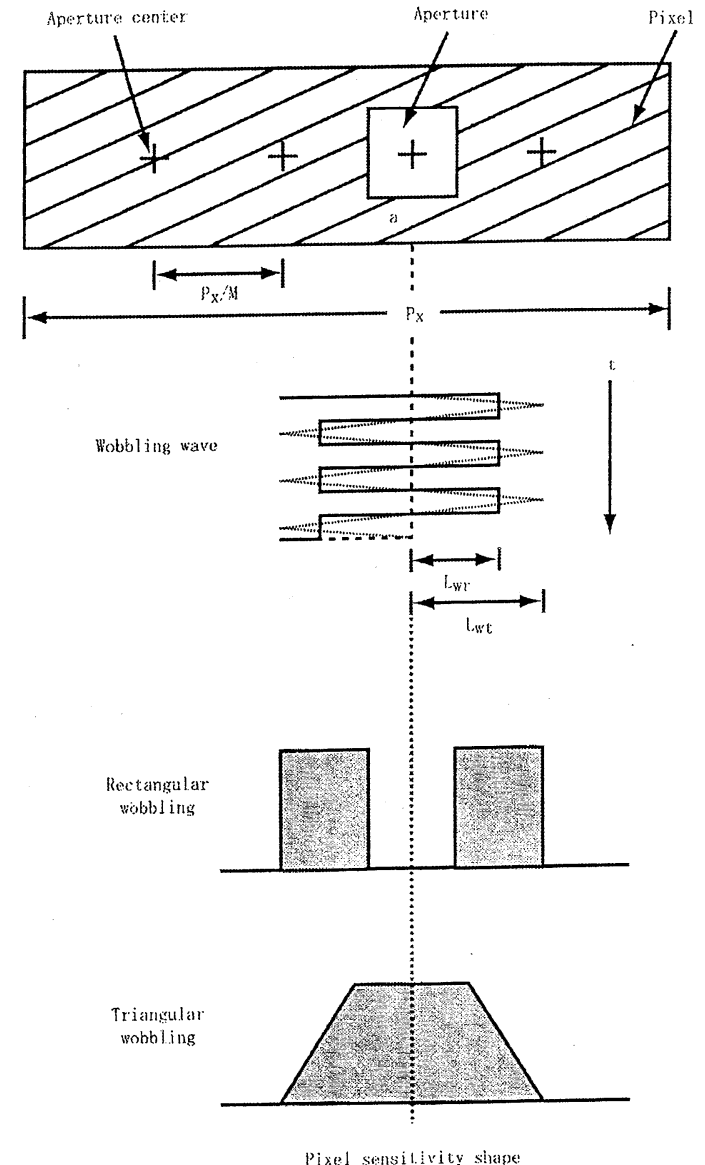

Fig. 9 The M-step wobbling operation with a rectangular and a triangular vibration wave, and the sensitivity shapes for image sensors or the brightness shapes for display devices.

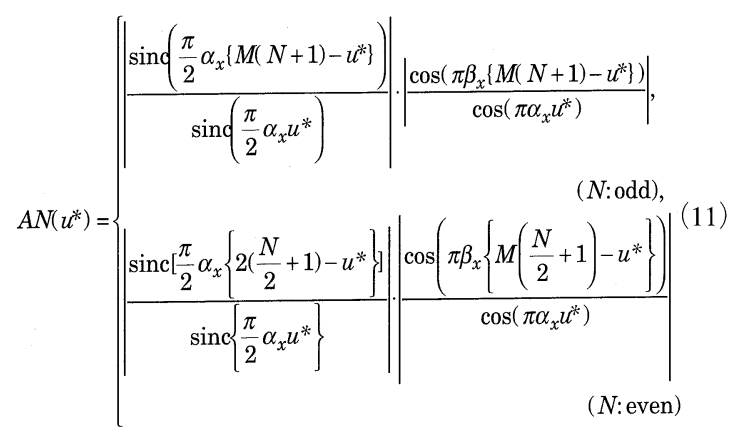

When $\alpha_{x}$ is $2 / M$, a double aliasing zero pinning is realized at $u^{*}=0$ and $M$. In this situation, it is required that the Nyquist frequency should be maintained to $M$ for high resolution. This requirement is satisfied by

$\cos \left(\pi \beta_{x} u^{*}\right) \geq 0,\left(0 \leq u^{*} \leq M\right)$

From this relationship, we obtain

Also, the double aliasing pinning can be realized by the

$$
\beta_{x} \leq \frac{1}{2 M}
$$

other condition;

$$
\beta_{x}=1 / 2 M, \alpha_{x}=1 / M
$$

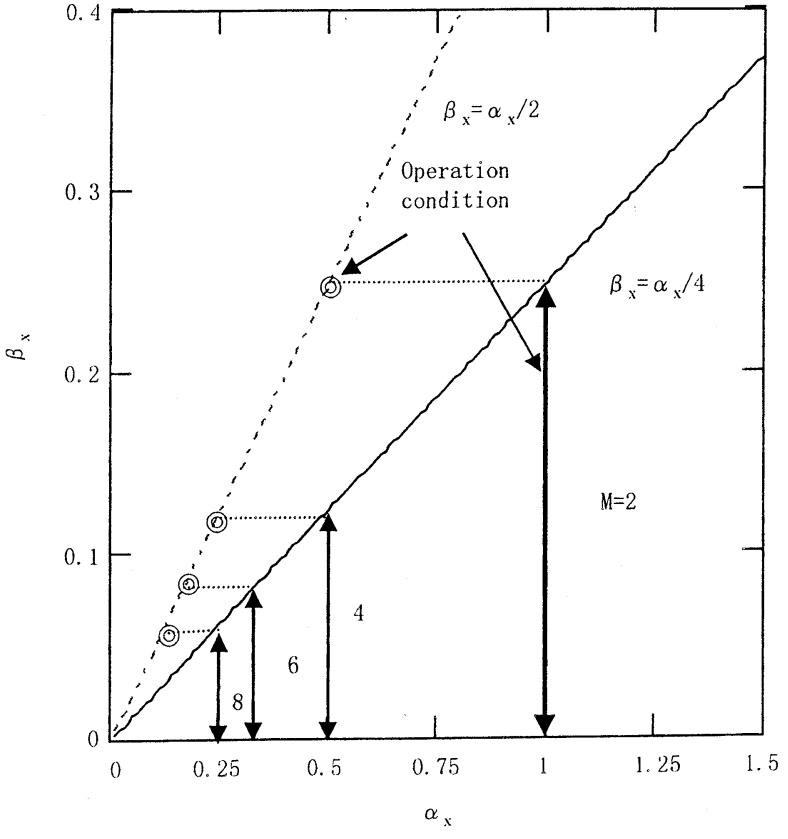

Fig.10 The optimum operation condition for the M-step rectangular wobbling.

The optimum operating condition, considering the double aliasing zero pinning and the short time aliasing compensation between two successive field, is shown in Fig. 10. As shown in this figure, high resolution with low aliasing is realized by setting appropriate $\alpha_{x}$ and $\beta_{x}$.

In the case of odd step swing operation, there is no counter field with $180^{\circ}$ phase difference. The pitch of Moire pattern, which appears at individual field, is given by the lowest common multiple between pitches of the bar pattern and the pixel ${ }^{10)}$. When the Moire frequency is denoted by $\mathrm{fm}$, the aliasing signal $A(\mathrm{k}, \mathrm{x})$ with a phase difference $\mathrm{k}(2 \pi / M)$ is given by

$$
A(k, x)=A_{0}+B_{0} \cos \left(2 \pi f_{m} x+\frac{2 \pi k}{M}\right)
$$

where $A_{0}$ and $B_{0}$ are an average amplitude and an aliasing variation amplitude, respectively. If $M$ is shown by $(2 m+1)$ where $m$ is integral, the aliasing signals with a spatial phase shifting of $2 \pi /(2 m+1)$ appear in a frameperiod, as shown in Fig.11. Combining these aliasing waves, an average signal $A A(m, x)$ by the $M$-step swing operation is obtained as follows;

$$
\begin{aligned}
A A(m, x) & =\frac{1}{M} \sum_{k=0}^{2 m}\left\{A_{0}+B_{0} \cos \left(2 \pi f_{m} x+k \frac{2 \pi}{2 m+1}\right)\right\} \\
& =A_{0}+\frac{B_{0}}{M} \sum_{k=0}^{2 m} \cos \left\{2 \pi f_{m} x+k \frac{2 \pi}{2 m+1}\right\}
\end{aligned}
$$




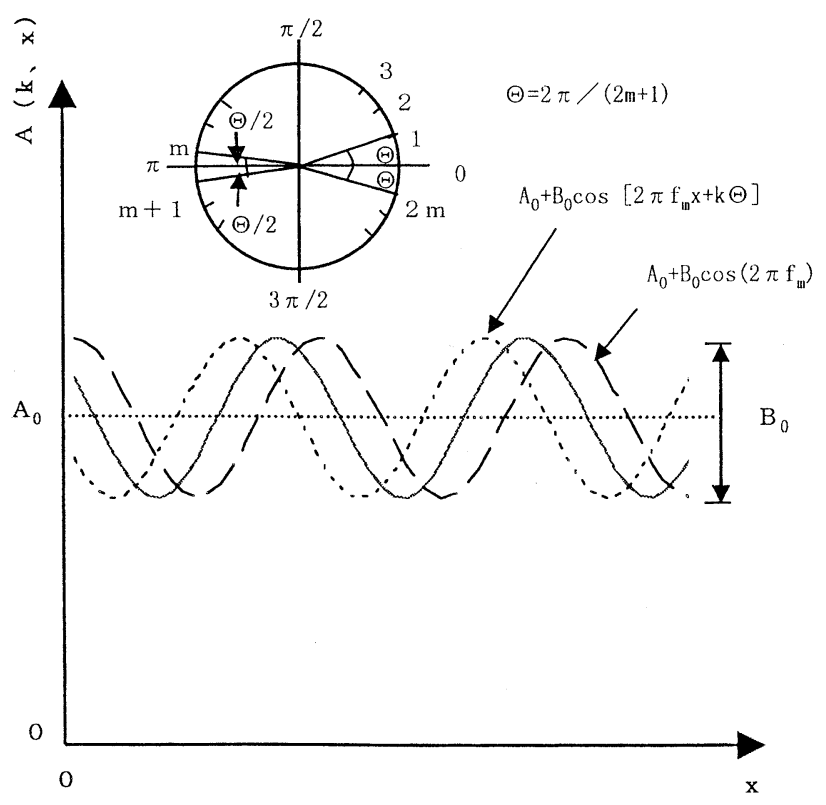

Fig.11 The aliasing signals for the M-step swing and wobbling operation.

$$
\begin{aligned}
= & A_{0}+\frac{B_{0}}{2 m+1} \sum_{k=0}^{2 m}\left\{\cos \left(2 \pi f_{m} x\right) \cos \left(k \frac{2 \pi}{2 m+1}\right)\right. \\
& \left.-\sin \left(2 \pi f_{m} x\right) \sin \left(k \frac{2 \pi}{2 m+1}\right)\right\} \\
= & A_{0}+\frac{B_{0}}{2 m+1}\left[\cos \left(2 \pi f_{m} x\right) \sum_{\mathrm{k}=0}^{2 m} \cos \left(k \frac{2 \pi}{2 m+1}\right)\right. \\
& \left.-\sin \left(2 \pi f_{m} x\right) \sum_{\mathrm{k}=0}^{2 m} \sin \left(k \frac{2 \pi}{2 m+1}\right)\right]
\end{aligned}
$$

The finite series of the trigonometric functions in Eq. (15) are

$$
\begin{aligned}
\sum_{k=0}^{2 m} \cos \left(k \frac{2 \pi}{2 m+1}\right) & =1+\sum_{k=1}^{m} \cos \left(k \frac{2 \pi}{2 m+1}\right)+\sum_{k=m+1}^{2 m} \cos \left(k \frac{2 \pi}{2 m+1}\right) \\
& =1+2 \sum_{k=1}^{m} \cos \left(k \frac{2 \pi}{2 m+1}\right) \\
& =1+2 \sin \left(\frac{m+1}{2 m+1} \pi\right) \cdot \sin \left(\frac{m}{2 m+1} \pi\right) / \sin \left(\frac{1}{2 m+1} \pi\right) \\
& =0
\end{aligned}
$$

and

$$
\begin{aligned}
\sum_{k=0}^{2 m} \sin \left(k \frac{2 \pi}{2 m+1}\right) & =\sum_{k=1}^{m} \sin \left(k \frac{2 \pi}{2 m+1}\right)+\sum_{k=m+1}^{2 m} \sin \left(k \frac{2 \pi}{2 m+1}\right) \\
& =0
\end{aligned}
$$

From these relationships, we obtain

$$
A A(m, x)=A_{0}
$$

This means that, in the case of odd step swing operation, the aliasing is finally suppressed at the end of the frame period as there is no counter field with $180^{\circ}$ phase difference. Therefore, the short time aliasing compensation cannot be realized.

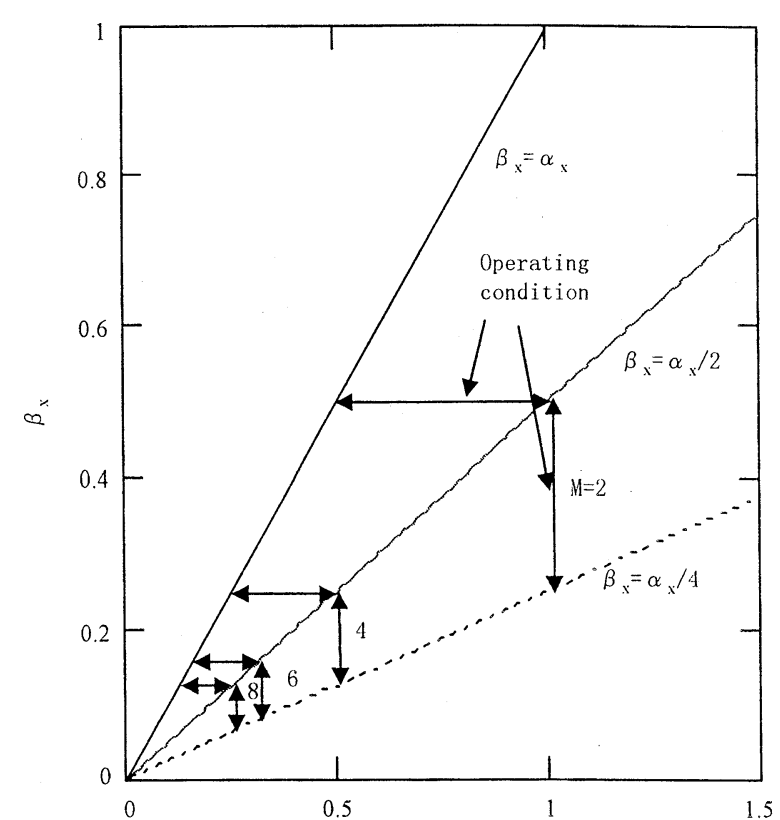

Fig.12 The optimum operation condition for the M-step triangular wobbling.

\subsection{Analysis on Triangular Wobbling}

The triangular wobbling operation can give a high resolution with lower aliasing and with wider operating condition than are attainable the rectangular wobbling. The MTF is shown by Eq. (8), in which the Nyquist frequency is $M$. Also, the Nth aliasing rate is given by

$$
A N\left(u^{*}\right)=\left\{\begin{array}{c}
\left|\frac{\operatorname{sinc} \frac{\pi}{2} \alpha_{x}\left\{M(N+1)-u^{*}\right\}}{\sin \left(\frac{\pi}{2} \alpha_{x} u^{*}\right)}\right|\left|\frac{\operatorname{sinc}\left[\pi \beta_{x}\left\{M(N+1)-u^{*}\right\}\right]}{\operatorname{sinc}\left(\pi \beta_{x} u^{*}\right)}\right| \\
\sin \left(\frac{\pi}{2} \alpha_{x}\left\{M\left(\frac{N}{2}+1\right)-u^{*}\right\}\right)|\cdot| \frac{\sin \left(\pi \beta_{x}\left\{M\left(\frac{N}{2}+1\right)-u^{*}\right\}\right)}{\sin \left(\frac{\pi}{2} \alpha_{x} u^{*}\right)}|| \frac{\operatorname{sinc}\left(\pi \beta_{x} u^{*}\right)}{(N: \text { oven })} \mid
\end{array}\right.
$$

when $a_{x}$ is set at $2 / N$, the double aliasing zero panning is obtained in the similar way as the rectangular wobbling. For the requirement of Nyquist frequency maintaining at $u^{*}=M, \beta_{x}$ satisfies a condition;

$$
\operatorname{sinc}\left(\pi \beta_{x} M\right) \geq 0, \quad\left(0 \leq u^{*} \leq M\right)
$$

Thus, we obtain

$$
\beta_{x} \leq \frac{1}{M}
$$

The aliasing rate at $\beta_{X}=1 / \mathrm{M}$ matches with that at $\beta_{x}=1 / 2 M$. The more $\beta_{X}$ increases at $\beta_{X} \leqq 1 / 2 M$, the less the aliasing rate monotonously decreases. Lower aliasing condition is obtained in the range as follows;

$1 / 2 M \leq \beta_{x} \leq 1 / M$ 
The optimum operating conditions for $M=4$ are obtained in Section 3 about the four step wobbling with triangular vibration wave, and that for $M=2$ was previously shown by the authors ${ }^{4)}$. Under the same consideration, lower aliasing condition of $\alpha_{x}$ in the case of the double aliasing zero pinning by $\beta_{x}=1 / M$ is obtained as follow

$$
1 / M \leqq \alpha_{x} \leqq 2 / M
$$

Fig.12 shows the optimum operating conditions for the multiple-step wobbling for high resolution with the double aliasing zero pinning and the short time aliasing suppression. As shown in this figure, the triangular wobbling gives high-resolution picture with lower aliasing and in wider operation range.

\section{Multiple-Step Swing High-Resolution Display Method for the Multiple-Step Swing Imaging Signals}

The multiple-step swing operation can be applied to a resolution enhancement for display devices as well as for image sensors. For a system with both multiple-step swing image sensor and display, suitable operation conditions are required for the high-resolution display. In the swing display method, display device is shifted synchronously with the signals of image sensor. Also the display pixel shifting can be realized by the Optical Super Resolution ( OSR ) method ${ }^{11)}$, using several optical plates settled on the display device. Fig.13 shows a multiple-step swing system construction. High-response display devices, such as a digital mirror device (DMD) ${ }^{12)}$ and an antiferroelectric (AF) - LCD ${ }^{13)}$, etc., are suitable for the swing operation because signals for all pixels must be rewritten during the vertical blanking period. For example, using DMD, digital reflection-light signals produced by changing an angle of a micro-mirror in a unit pixel are projected on a distant screen. The pixels in the DMD are periodically arranged in two dimensions. Accordingly, the pixels on the screen are also projected in the same arrangement on the DMD. After a field picture is displayed on the screen, picture signals of the next field are written in the DMD and displayed on the screen during the next field. The pixel positions are horizontally shifted in multiple-steps. The pixel shifting is achieved by moving a DMD chip, or by a projection image shifting using an optical equipment. Time-shared images horizontally shifted are displayed on the screen, resulting in high-resolution display.

The multiple-step swing display method is divided roughly into three modes, (1) the multiple-step swing imaging signals are individually displayed on the screen

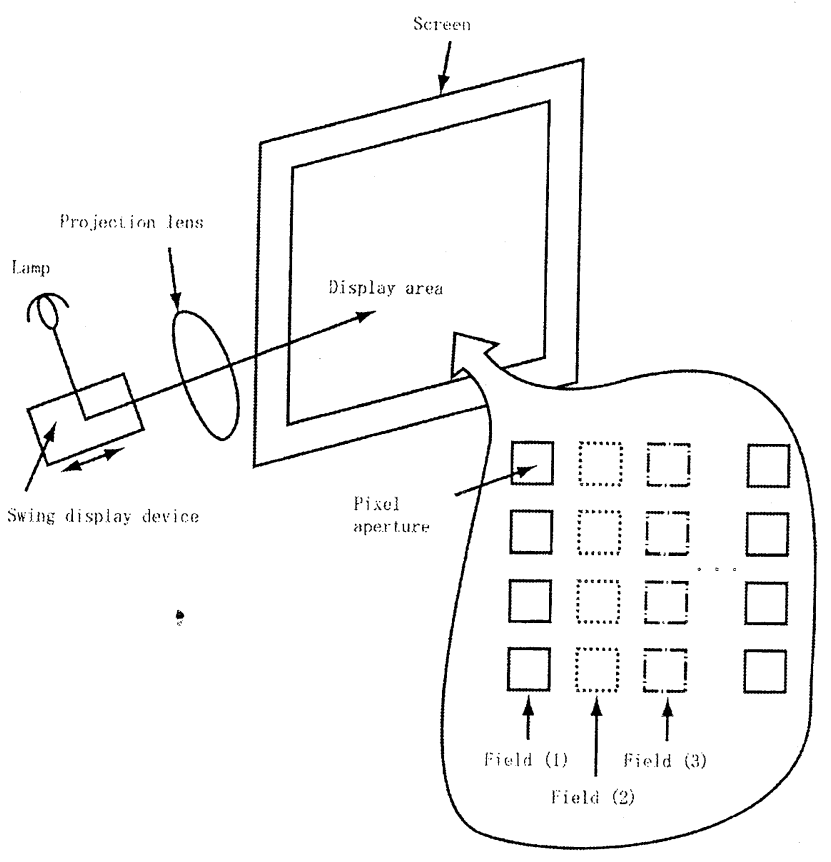

Fig.13 Swing operation application for a display device using DMD.

with the same step mode of the image sensor, (2) the imaging signals divided in two sections, using a frame memory, are displayed in two field periods, and (3) the imaging signals divided in multiple sections are displayed on the screen. The second method has an advantage in that it is applicable to the standard TV format with two fields, but needs pixel sampling data rearrangement using a frame memory with large capacity. For the third method, the construction of image sensor and display device is designed in a way based on a consideration of system requirements and device performance.

Fig.14 shows pixel sampling points in an original one pixel region for the multiple-step swing operation. For the $M$-step swing operation, the pixel sampling points are arranged with a $p_{x} / M$ pitch. $M$ is an even number in order to realize the short time aliasing compensation by setting two successive fields with $180^{\circ}$ phase difference. As shown in this figure, the pixel sampling points of odd and even fields are arranged separately into two areas, odd field area and even field area, in the original one pixel region. In the first method in which each pixel is individually displayed, the counter pair fields for the short time aliasing compensation are arranged in time series. For the second method with two field display formats, the total aliasing compensation is finally achieved at the end of the two field periods. In this case, the number of pixels shared in each of the two fields must be the same. In addition, a display with lower field flicker can be obtained when each field is constructed by the counter 


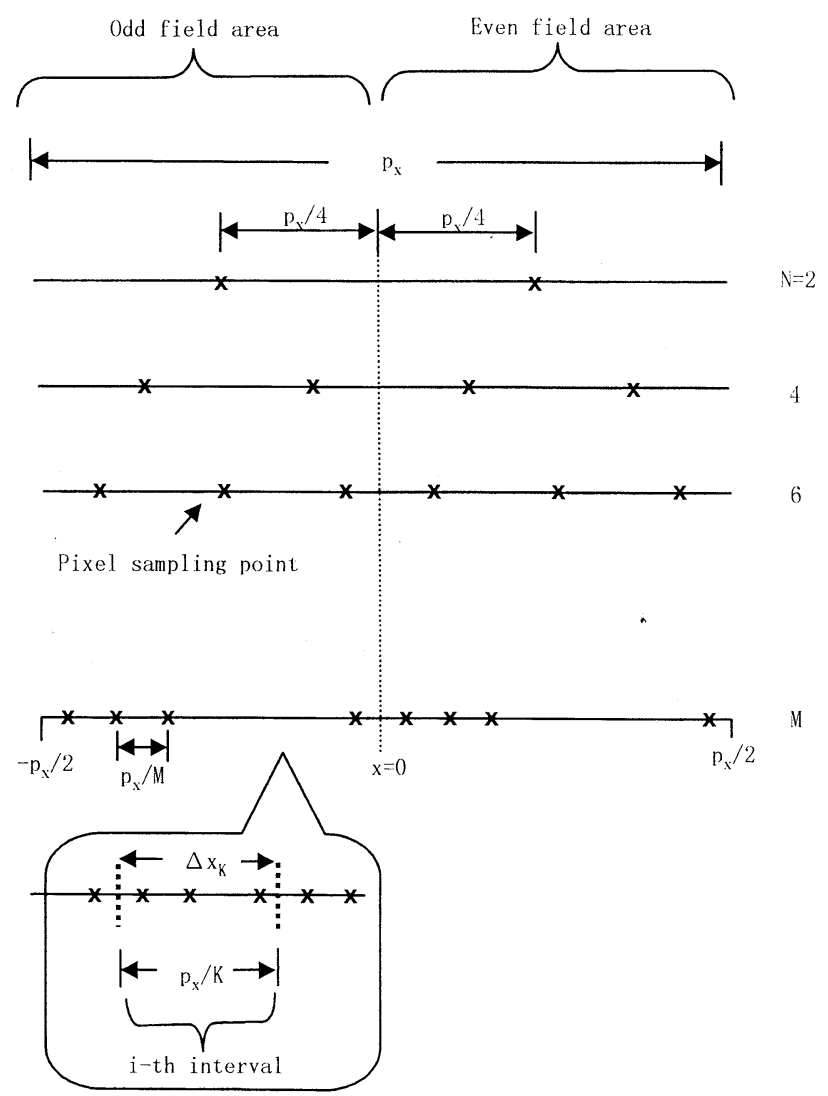

Fig.14 Pixel sampling points in an original one pixel region for the multiple-step swing operation.

pairs, because the main short time aliasing compensation is realized in one field period. Considering $K$-step swing display operation in which $K$ is less than $M$, an optimum operation condition is required. Pixel sampling function $F_{M}(x)$ is written by the sum of pixel sampling function at the odd field area $F_{o}(x)$ and that at the even field area $F_{e}(x)$ as follows

$$
\begin{aligned}
F_{m}(\mathrm{x})= & F_{0}(\mathrm{x})+F_{e}(\mathrm{x}) \\
= & \sum_{n=0}^{M / 2^{-1}} \delta\left(x+\frac{2 n+1}{2} \cdot \frac{p_{x}}{M}\right)+ \\
& \sum_{n=0}^{M / 2^{-1}} \delta\left(x+\frac{2 n+1-M}{2} \cdot \frac{p_{x}}{M}\right)
\end{aligned}
$$

Pixel sampling points of $M$ number are divided into number of $K$. Number of pixel sampling points in the unit interval $\Delta x_{K}$ is $M / K$. In this case, $M / K$ must be integer for the pixel arrangement with the same pixel number in each interval. $K$ must be also even for the short time aliasing compensation. Considering the short time aliasing compensation, pixel sampling function fi $(x)$ of two successive fields is written as follows:

$$
\begin{aligned}
f_{i}(x)= & \sum_{n=0}^{M / K^{-1}} \delta\left(x+\frac{i-1}{K} p_{x}+\frac{2 n+1}{2} \cdot \frac{p_{x}}{M}\right)+ \\
& \sum_{n=0}^{M / K} \delta\left(x+\frac{i-1}{K} p_{x}+\frac{2 n+1-M}{2} \cdot \frac{p_{x}}{M}\right)
\end{aligned}
$$

And, $F_{M}(x)$ is given by

$$
F_{M}(x)=\sum_{n=0}^{M / 2-1} f_{i}(x)
$$

In this display system, it is desirable for the image sensor with the $M$-step swing operation to sample successively in the unit interval to reduce the frame memory capacity used in the system. As mentioned above, the multiple-step swing display method is derived for the resolution enhancement .

\section{Conclusion}

Resolution improvement is strongly required for solidstate image sensors. To satisfy this requirement, we analyzed a novel multiple-step swing and wobbling operation with a view to applying it as a higher resolution method for solid-state image sensors. We derived optimum operation conditions for the high resolution, taking account of low aliasing through the analysis. It is important to hold the aliasing at $u^{*}=0$ and Nyquist frequency to be zero for the resolution enhancement with better picture quality. For effective aliasing suppression, the number of steps should be even because the aliasing can be compensated by combining two counter fields having $180^{\circ}$ phase difference. In addition, the short time aliasing compensation in a two field period is realized by setting two successive fields with $180^{\circ}$ phase difference. We derived that a multiple-step wobbling operation with the triangular wave realized high resolution with lower aliasing, and further derived the optimum operation condition of the pixel aperture ratio $\alpha_{\mathrm{x}}$ and the wobbling rate $\beta_{x}$. The multiple-step swing operation can be applied for the resolution enhancement of display devices. Suitable display conditions of the multiple-step swing imaging signals were derived for achieving high resolution display images.

The resolution enhancement technique using the multiple-step swing and wobbling operation will be able to open a path not only to the next-generation ultra-highresolution solid-state image sensors but also to similar display devices. 


\section{[References]}

1) N.Harada, Y.Endo, Y.Hayashimoto, C.Egawa, C.Tanuma, K.Yokoyama and O.Yoshida : "A Swing CCD Image Sensor", SID Japan Display 83 Digest of Technical Papers, pp.424-427 (1983)

2) O.Yoshida, Y.Endo, Y.Egawa, Y.Hayashimoto, C.Tanuma, K.Yokoyama and N.Harada : "High Resolution CCD Image with CCD-Chip-Shift Operation", J. of Imaging Technology, 11, 5, pp.205-209 (1985)

3) K.A.Hoaggland : "Image-Shift Resolution Enchancement Techniques for CCD Imagers", SID International Symposium Digest of Technical Papers, 288 (1982)

4) N.Harada and O.Yoshida : " Analisis on High Resolution Method for CCD Image Sensor Using Swing and Wobbling Imaging", J. of The Institute of Image Information and Television Engineers, 55, 2, pp.317-324 (2001)

5) S.Habara, S.Teraoka, T.Tanuma, H.Honda, Y.endo, N.Harada : "Synchro Vision CCD Image Sensor", Technical Report of The Institute of Television Engineers of Japan, 10,4, pp.53-58 (1986)

6) O.Yoshida and A.Iwamoto : "Two Dimensional Nyquist Limit and MTF for Solid State Image Sensor", The Journal of The Institute of Television Engineers of Japan, 37, 10, pp.819-825 (1983)

7) N.Harada, S.Uya, Y.Hayasimoto, Y.Endo, T.Adachi, O.Yoshida : "A 1/2" Format Two-Level CCD Imager with $492 \times 800$ Pixels", ISSCC 85, Digest of Tech. Papers, XVII, FAM18.6, pp.270-271 (1985)

8) S.Manabe, Y.Maunaga, A.Furukawa, K.Yano, Y.Endo, N.Harada: "A 2Million-Pixel CCD Image Sensor Overlaid with an Amorphous Silicon Photoconversion Layer", IEEE Trans. Electron Devices, ED-38, 8, pp.17651770 (1991)

9) O.Yoshida, Y.Endo,Y.Egawa and N.Harada : "Image Flicker Control by CCD-Chip-Shift and Wobble Operation at Double-Enhanced Nyquist Regions", IEEE Trans. Electron Devices, ED-32, 8, pp.1505-1510 (1985)

10) O.Yoshida and A.Iwamoto : " Moire Patterns on Video Pictures Taken by CCD Image Sensors", Technical Report of The Institute of Television Engineers of Japan, IE80-89, pp.9-16 (1987)

11) T.Togino : "The latest technical trend and the view of HMDs", Technical Report of The Institute of Image Electronics Engineers of Japan, 00-04-02 (2000)

12) L.J.Hornbeck : "Current Status of the Digital Micromirror Device (DMD) for Projection Television Application", IEDM 93, Digest of Technical Papers, pp.381-384 (1993)

13) T.Yoshida, T.Tanaka, J.Ogura, H.Wakai, H.Aoki : "A Full-Color Thresholdless Antiferroelectric LCD Exhibiting Wide Viewing Angle with Fast Response Time", SID 97, Digest of Technical Papers, pp.841-844 (1997)

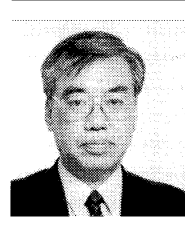

\section{Nozomu Harada}

He was born in Kagawa, Japan, on August 25, 1945. He received the B.S., M.S., and Doctor degrees in electrical engineering from Shizuoka University, Japan, in 1968, 1970, and 2001, respectively. In 1970, he joined the R \& D Center, Toshiba Corporation, where he engaged in research and development of high-sensitivity image tubes and CCD image sensors. In 1984, he joined the Toshiba ULSI Research Center, where he worked on the development of solid-state image sensors and managed developments of DRAM, Flash EEP ROM and GaAs IC. Since 1993, he has been engaging in the developments of poly-Si TFT-LCD and other advanced LCD technologies in the Toshiba Display Device engineering Laboratory and the LCD R \& D Center, respectively. He then moved Toshiba Electronics Engineering Corporation in 2000 and has been working on the development of LCD design technologies. He received Suzuki Award and NiwaTakayanagi Award from the Institute of Television Engineers of Japan in 1973 and 1984, respectively. He received two Invention Awards from the Japan Institute of Invention and Innovation in 1990 and 1991, respectively. He also received Best Paper Award from Institute of Electrostatics of Japan in 1998. Dr.Harada is a member of the Institute of Image Information and Television Engineers, the Japan Society of Applied Physics, and Japan Ergonomics Society.

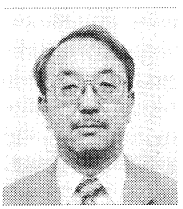

\section{Okio Yoshida}

He was born in Tokyo, Japan, on September 15, 1939. He received the B. E., M.E., and Doctor degrees in electronics engineering from Tohoku University, Sendai, Japan in 1962, 1964, and 1973, respectively. Since 1964 he joined the R\&D Center, Toshiba Corporation, Kawasaki, Japan and engaged in research and development of photoelectronic imaging devices-first vidicon-type camera tubes and later CCD imaging devices at the ULSI Research Laboratories, Toshiba Corporation. He then moved to the Technology Planning Coordination Division of the Principal Office and was dispatched to the Toshiba London Office as Toshiba Technology Representative for Europe from 1985 to 1989 . Returning to Japan, he joined again the Laboratories and the Division. He retired form Toshiba Corporation in 2000 after having worked at the Corporate Planning Division and the International Relation Division. He is a professor of Department of Media and Culture Studies at Josai International University from 2001. He is a Fellow of IEEE, a Fellow of IEE and a Chartered Engineer, a member of IEICE of Japan, IEICE of Japan and JSAP. 